\title{
Quantification of Dependencies in Electrical and Information Infrastructures: the CRUTIAL approach*
}

\author{
Marco Beccuti, Giuliana Franceschinis \\ Dip. di Informatica, Univ. del Piemonte Orientale, Italy \\ \{beccuti, giuliana\}@mfn.unipmn.it \\ Silvano Chiaradonna, Felicita Di Giandomenico \\ ISTI Department, CNR, Italy \\ \{chiaradonna, digiandomenico\}@isti.cnr.it
}

\author{
Susanna Donatelli \\ Dip. di Informatica, Univ. di Torino, Italy \\ susi@di.unito.it \\ Paolo Lollini \\ Dip. di Sistemi e Informatica, Univ. di Firenze, Italy \\ lollini@unifi.it
}

\author{
Giovanna Dondossola, Fabrizio Garrone \\ Power Systems Development, Department CESI Ricerca, Italy \\ \{dondossola,garrone\}@ cesiricerca.it
}

\begin{abstract}
In this paper we present the CRUTIAL approach to model and quantify (inter)dependencies between the Electrical Infrastructure (EI) and the Information Infrastructures (II) that implements the EI control and monitoring system. The quantification is achieved through the integration of two models: one that concentrates more on the structure of the power grid and its physical quantities and one that concentrates on the behaviour of the control system supported by the II. The modelling approach is exemplified on a scenario whose goal is to study the effects of a II partial failure (a denial of service attack that compromises the communication network) on the remote control of the EI.
\end{abstract}

\section{Introduction}

This paper describes the approach that has been pursued in the European project CRUTIAL [9] to use stochastic modelling techniques to model and quantify interdependencies in the Electrical Power Systems (EPS). The main challenge for CRUTIAL is to make power control resilient in spite of threats to their information and communication infrastructures. Considering the crucial role of control systems in governing the quality and the stability of the electric power service, it is considered of great importance for the utilities operating the infrastructures to have tools for analysing threat impacts and technologies for avoiding, or

\footnotetext{
*Acknowledgements: This work has been partially supported by the European Commission (project IST-4-27513 CRUTIAL)
}

limiting, most serious consequences. The project focuses on the electrical infrastructure (EI) and the information infrastructures (II), by considering different topology realms and different kinds of risk. CRUTIAL addresses a number of problems in the field, in particular architectural solutions have been devised to make EPS more resilient to cyber attacks and testbeds have been implemented to check the proposed solutions on contexts that emulate, on a necessarily small scale, the EPS behaviour. In order to master the complex mechanisms of global failures, particular focus was put on the study and modelling of the types of failures that are characteristic of interdependent critical infrastructures. Although the modelling of such failures has received increasing interest in the last years after the large blackouts of electric power transmission systems in 1996 and 2003, there is still no definite understanding on EPS interdependencies, and on the techniques to evaluate the the impact of cascading, escalating and common cause failures.

The effort of CRUTIAL has addressed a number of scenarios, mainly patterns of interaction between II and EI. In this paper the most critical scenario described in [13] has been selected for experimenting with the CRUTIAL Modelling Framework. This scenario explores the security of the communications between the Transmission and Distribution System Operators under emergency operating conditions, assessing the possible cascading effects of ICT threats to the communication channels connecting the TSO and the DSO Control Centres (TSO CC and DSO CC respectively) and the Substation Monitoring Control and Defence Terminal Units (MCD-TUs). Cyber attacks carried out under emergency conditions, when defence actions have to be performed under strict real time constraints, can cause se- 
vere damages, like inhibiting the proper execution of the required automatic load shedding actions. Load reduction usually occurs when the power system is exposed to disturbances due to deficiency conditions (faults, loss of generation, switching errors, etc) [8]. Major disturbances may have a dramatic impact on the performance of the power system, requiring fast and reliable load shedding actions which must be set and timed properly: inappropriate load reduction caused by the execution of an improper load shedding scheme may be inefficient and even cause cascading effects.

Modelling effort in CRUTIAL and paper contribution. The modelling framework addressed by CRUTIAL [9] includes both qualitative and quantitative analysis and evaluation methods. Qualitative models have been defined that capture the interaction of EI and II [17] and that have led to the definition of a new class of automata [12]. Qualitative modelling will not be discussed in this paper.

To provide a quantitative evaluation of the impact of II failure on the global EPS behaviour two main approaches have been pursued. The first one is based on an integration of a Stochastic Activity Network (SAN) [10, 18] model of the EPS with techniques imported from the power engineering field to model and simulate the electrical state of the EPS. This approach (that we shall call SAN for short) allows a rather detailed model of the structure of the EPS, although the model of the control algorithms and of the counter measure that takes place upon a failure are treated at a rather abstract level. The second one is based on Stochastic Well-formed Net (SWN) [2, 14] and is more centred around the protocols addressed by the scenario, so it can represent in some detail the scenarios, but it has to make stochastic assumptions on the EI behaviour.

Previous literature. Understanding the reciprocal effects of interdependencies among interacting critical infrastructures, as well as quantifying resiliency, security and robustness related indicators are tackled by a number of research initiatives/organisations. An overview is provided in [5]. In addition to the work performed by the CRUTIAL consortium (partly represented by these authors), several models based on simulation have been proposed to represent Electric Power Systems [1, 4, 11]. These studies mainly focus on reproducing network disruptions, which eventually lead to blackouts, in order to estimate the vulnerabilities of the system or the impact on the EPS reliability of important network parameters, such as overload or load demand, in presence of disruptions. In most of these approaches, the modelling of the existing SCADA systems and of II control is not considered explicitly or is very simple. The ongoing European project IRRIIS [15] devotes significant effort to interdependencies analysis and modelling. The IRRIIS consortium is developing SimCIP [19], an integrated simulation environment used for the modelling and simu- lation of critical infrastructures' interdependencies, based on the ISE (Implementation, Services and Effects) metamodel [3]. Both electric power and telecommunication networks are considered. The modelling framework addressed by CRUTIAL [9] includes both qualitative and quantitative analysis and evaluation methods, and is aimed at building generic models of interdependencies $[6,7,16,17]$. In addition, two testbeds are under development [13].

\section{The considered scenario}

The scenario modelled in this paper considers a situation in which a load shedding activity is needed to re-establish the EI working conditions upon an electrical failure, but the II is not working properly due to a Denial of Service (DoS) attack on the communication channel between DSO CC and the DSO Substations (DSO SSs). In emergency conditions the TSO is authorised by the DSO to activate load shedding activities on the Distribution Grid. The TSO CC monitors the Electric Power System and detects some potentially dangerous conditions that could be recovered with appropriate load shedding commands applied to particular areas of the Grid. In order to actuate this defence action the TSO CC chooses a subset of DSO SSs from the list of DSO SSs participating in the emergency plan, then sends the requests of preventively arming the MCD-TUs of these SSs to the interested DSO CC. These requests are delivered through communication channels between TSO CC and DSO CC. The DSO CC forwards the arm command to the required SSs, and returns their status to the TSO CC. If the potential emergency condition evolves into a real emergency situation, the TSO SS sends the load shedding command to all the DSO SSs participating to the emergency plan, but only the substations that have been previously armed will be actually detached. TSO SS periodically sends test packets toward detachable substations. If an armed substation does not receive the expected test packet within $1 \mathrm{~min}$, it automatically disarms itself. Disarming also occurs after 20 min. from the arming command if no load shedding command is issued.

Depending on when the Substation DoS occurs in this scenario evolution, different behaviours may be envisaged. A DoS attack starting before issuing the arming command towards a given substation creates the possibility of preventing the execution of that substations trip. A DoS attack takes place when the substation is armed, the attack denies the successful execution of the periodic testing and causes the consequent automatic disarming of the substation. Finally the DoS may occur just before issuing the substation trip thus denying the possibility of defending the system from extreme contingencies. The effects on the EPS of the considered DoS will depend on the number of components attacked and on the pattern and intensity of the DoS. The 
identification of the dependencies of EI and II upon an electrical failure in presence of a DoS, and their quantification, is the aim of this paper.

\section{The SWN model}

The SWN models in CRUTIAL focus on the ICT control system implemented by the II infrastructure. The model presented in the sequel represents the automation system behaviour under a DoS attack depicted in the scenario described in Section 2. In Fig.1A a SWN model of the scenario is depicted: it represents in an abstract way the event causing the unbalance in the EI that triggers the arming and the load shedding procedure (transitions e-failure, StartArmingProcess, EndArmingProcess, LoadShedding).

The TSO CC is represented in an abstract way by two places and three transitions: ProcInfo and ReadyRqArming together with transitions StartArmingProcess, EndArmingProcess and TransmitArming. A token in place ProcInfo means that the TSO CC is analyzing the EI and elaborating some potential emergency conditions that could be fixed with suitable load shedding commands applied to particular areas of the EPS. When the defence actions have been elaborated (transition StratArmingProcess), TSO CC is ready to actuate them; this requires to send a request of preventively arming some SSs to the DSO CC (transition TransmitArming). The DSO CC sends the arming command to the appropriate substations (sequence CC_selection, ForwardedPacket and TxDelay): at this point the influence of the DOS attack (if it has started) is modelled by the choice between transitions ForwardedPacket and LostPacket. The firing probability of these two transitions depends on the progress level of the DOS attack, represented by the marking of place AttackSeverity (which in turn is modified by transition IncreaseSeverity while place Active is marked). If the packet is forwarded, its transmission time can still depend on the progress of the DOS attack. If the message is forwarded to the substation, the latter is armed (place SS_armed), moreover it sends back a message to the DSO $\mathrm{CC}$ (the return message is subject to the same risk of being lost in case a DOS attack is ongoing, modelled by transitions LostPacket and ForwardedPacket).

When a perturbation in the EI is detected (place Partial e-outage marked), a load shedding command is sent from the TSO SS (called TSO sentinel) to (a subset of) the armed DSO SSs (transition LoadShedding): those that were armed, upon reception of the load shedding command perform the required procedure. If enough substations complete such procedure, the whole process ends successfully (firing of transition e-restoration ) and the $\mathrm{EI}$ is brought back in a new balanced state (place $e$-working). An arming command not followed by any load shedding, expires after some time (transition armedExpired). The DoS attack is represented in an abstract way by three places and three transitions: places Idle and Active together with transitions Begin_attack and End_attack model the presence/absence of an attack and the transitions from one state to the other; place AttackSeverity on the other hand models the status of the connection towards the attacked site(s): when the attack is ongoing, the connection capacity degrades (transition IncreaseSeverity) while the absence of attack restores the status back to normal. The DSO CC may detect that there is a communication problem with some substations when the acknowledge messages are not received within a certain time (transition Delay).

Observe that the transition \#Reset in the EI submodel is a special transition, with the following semantics. When it is enabled its firing brings the model back to the initial marking: the relative throughput of this transition with respect to the throughput of the TransmitArming transition provides an indication of the percentage of unsuccessful load shedding activities under a DoS attack. The particular semantics of the reset transitions allows this measure to be computed in steady state. For the SWN model of Fig. 1A to correctly represent the global behaviour of the EPS under a DoS attack, a number of parameters should be estimated, grouped in three sets. Parameters related to the "physical" scenario: how many substations, communication network between them, number of substations controlled by an area control center (and identification of the substations involved in an arming command or a load shedding one). Parameters related to EI: distribution or time value from arming and consequent load shedding command, and from arming to unarming. These times depend on the EI state. Parameters related to the II: distributions or time values related to the communication channel (e.g the package delay, probability of losing a packet) and to DoS attack (duration and severity of the attack).

Given a scenario where the first set of parameters is fixed, the third set could be part of a sensitivity analysis performed with the SWN model, while the second set depends from the first set in a way that is not explicitly modelled in the SWN model (since it requires to model the electrical state and its evolution).

\section{The SAN model}

To allow a realistic estimation of the impact of the DoS on the EI, we have decided to make use of the SAN modelling approach of CRUTIAL, reported in [7, 16], and here summarised in its general aspects and in those aspects that will be the base for the integration. The SAN approach has been developed in the context of the Transmission grid and uses a terminology that is slightly different form the one used for the scenario and SWN description. To allow the reader to refer to both the original paper [7] and the sce- 


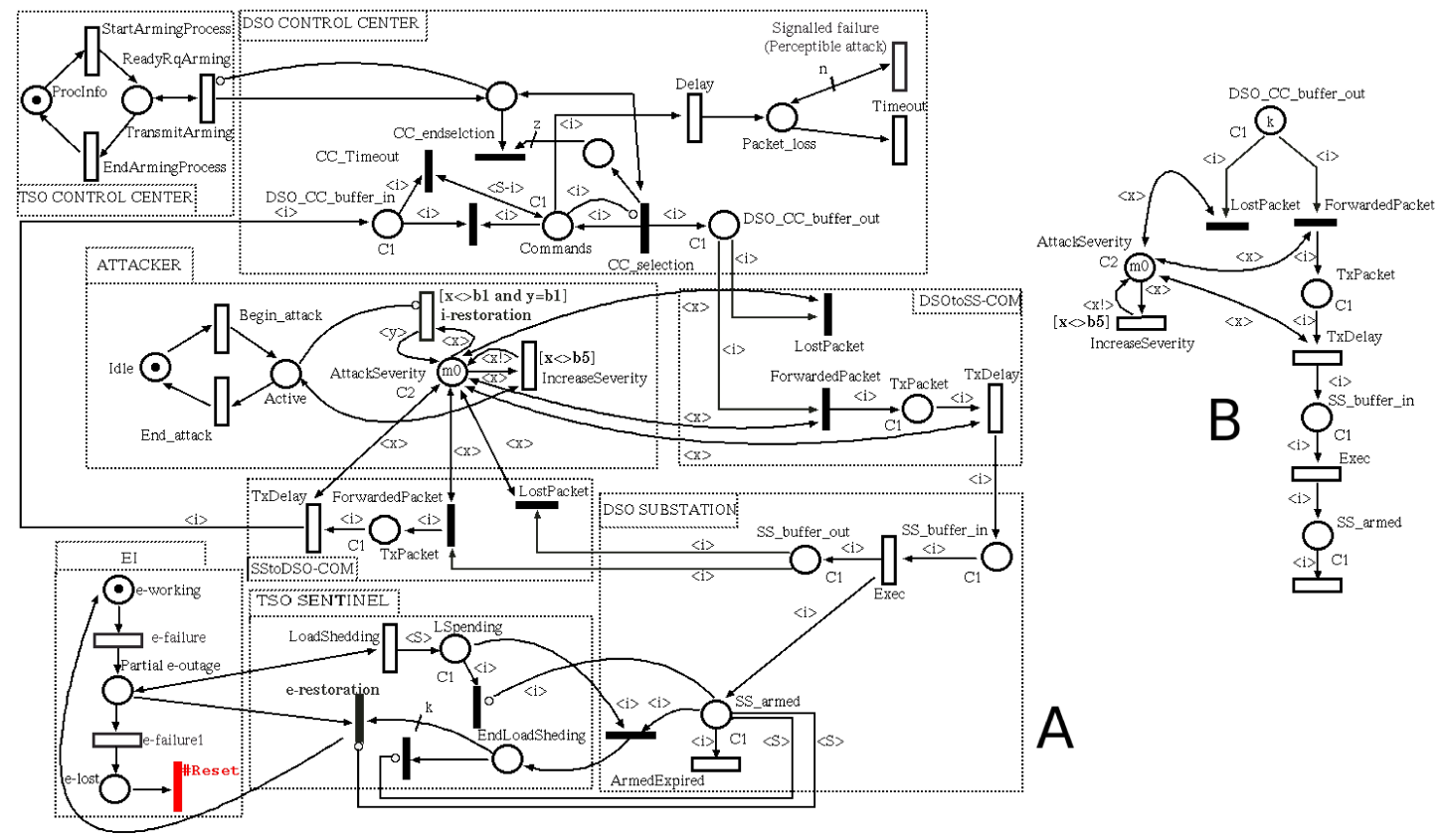

Figure 1: A) SWN reference model for scenario 2; B) Simplified SWN model for the interaction with the SAN model.

nario definition, we shall provide a mapping of the major terminology items.

Logical scheme of EPS. The EPS that we consider are limited to a homogeneous region of the transmission grid and to the corresponding regional control system. The logical structure is depicted in Fig. 2.

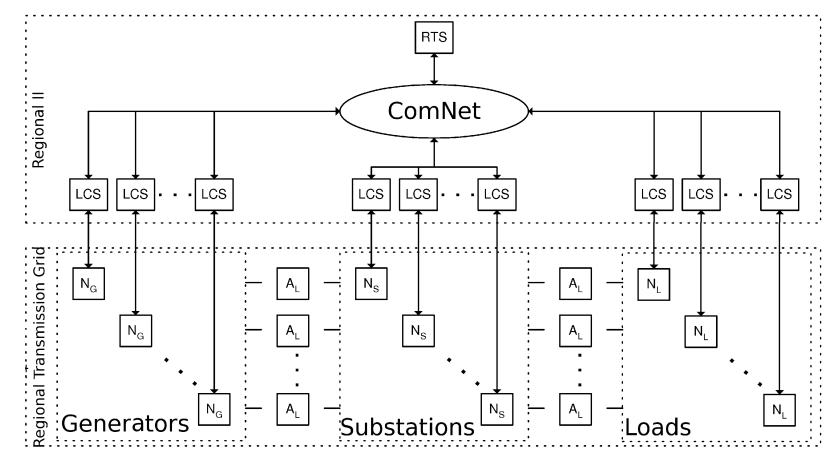

Figure 2: Logical structure of the analysed EPS instance

In the bottom part of Fig. 2 we can see the main elements that constitute the overall electric infrastructure, and thus in particular a region of the transmission power grid: generators ( $N_{G}$ components), substations ( $N_{S}$ components), loads ( $N_{L}$ components) and power lines $\left(A_{L}\right.$ components, which also logically include breakers and protections connected to the power lines). The energy produced by the generators is adapted by transformers, to be conveyed with min- imal dispersion, to the different types of end users (loads), through different power grids. The power lines are components that physically connect the substations with the power plants and the final users, and the substations are structured components in which the electric power is transformed and split over several lines. In the substations there are transformers and several kinds of connection components (like bus-bars, protections and breakers).

Some simplifying assumptions have been made to represent the power flow through the transmission grid, following the same approach used in $[1,4,11]$. Therefore, the state and the evolution of the transmission grid are described by the active power flow $F$ on the lines and the active power $P$ at the nodes (generators, loads or substations), which satisfy linear equations for a direct current (DC) load flow approximation of the $\mathrm{AC}$ system.

The Information Infrastructure implements the information control system managing the electrical grid. Among the several logical components composing II (all detailed in [7]), here we focus the attention on the teleoperation system for a region of the transmission grid (named TTOS), since its failure can affect a large portion of the grid, also leading to black-out phenomena. In the upper part of Fig. 2 we have depicted a possible logical structure of a regional II, i.e., the part of the information control system controlling and operating on a region of the transmission grid. The components $L C S$ (Local Control System) and RTS (Regional Telecontrol System) differ for their criticality and for the locality of their decisions, and they can exchange grid status infor- 
mation and control data over a (public or private) network (ComNet component). LCS guarantees the correct operation of a node equipment and reconfigures the node in case of breakdown of some apparatus. They include the acquisition and control equipment (sensors and actuators). $R T S$ monitors its assigned region in order to diagnose faults on the power lines. In case of breakdowns, it chooses the most suitable corrective actions to restore the functionality of the grid. Since RTS is not directly connected to the substations, the corrective actions to adopt are put in operation through the pertinent $L C S$. RTS corresponds to the TSO CC of the scenario, and $L C S$ corresponds to the MCD-TU. Note that the concept of area control center (TSO and DSO of the scenario) is not explicitly modelled. It will be part of the control function explained in the sequel $\left(R S_{1}\right.$ and $\left.R S_{2}\right)$ to determine which set of LCS are involved in a reconfiguration/load shedding action.

II controls the correct functioning of EI and activates proper reconfigurations in case of failure of, or integration of, repaired/new EI components. Such operations are not considered in detail but they are abstracted at two levels, on the basis of the locality of the EI state considered by II to decide on proper reactions to disruptions. Each level is characterised by an activation condition (that specifies the events that enable the II reaction), a reaction delay (representing the overall computation and application time needed by II to apply a reconfiguration) and a reconfiguration strategy $(R S)$, based on generation re-dispatch and/or load shedding. The reconfiguration strategy $R S$ defines how the configuration of EI changes when II reacts to a failure. For each level, a different reconfiguration function is considered: $R S_{1}($ ), to represent the effect on the regional transmission grid of the reactions of II to an event that has compromised the electrical equilibrium ${ }^{1}$ of EI, when only the state local to the affected EI components is considered. $R S_{1}()$ is performed by $L C S$ components and, because of the limited information necessary to issue its output, it is fast in providing its reaction. $R S_{2}()$, to represent the effect on the regional transmission grid of the reactions of II to an event that has compromised the electrical equilibrium of EI, when the state global of the whole EI system under the control of II is considered. Therefore, $R S_{2}()$ is determined on the global EI state and reacts in a longer time. When new events occur changing the status of EI during the evaluation of $R S_{2}()$, then the evaluation of $R S_{2}()$ is restarted based on the new topology generated by such events. $R S_{2}()$ is performed by RTS.

The activation condition, the reaction delay and the definition of the functions $R S_{1}()$ and $R S_{2}()$ depend on the policies and algorithms adopted by TTOS.

\footnotetext{
${ }^{1}$ Events that impact on the electrical equilibrium are typically an EI component's failure or the insertion of a new/repaired EI component; for simplicity, in the following we will mainly refer to failures.
}

In the current implementation, the output of $R S_{1}()$ is obtained by the solution (values for active power vectors $P$ and $F$ ) of power flow equations while minimising a simple cost function, indicating the cost incurred in having loads not satisfied and having the generators producing more power. The output values of $R S_{2}()$ for $P$ and $F$ are derived by solving an optimisation problem to minimise the change in generation or load shedding, considering more sophisticated system constraints. The reconfiguration strategy $R S_{1}()$ is applied immediately, while $R S_{2}()$ is applied after a time needed by $R T S$ to evaluate it. All these functions are based on the state of EI at the time immediately before the occurrence of the failure.

SAN model of EPS. The logical EPS scheme just presented has been modelled through the SAN formalism [18] a generalisation of Stochastic Petri Nets featuring state variables of any type (including doubles) and C-like functions to express transitions' enabling and state change.

We recall here the composed SAN model representing the overall considered EPS instance. The following atomic models have been identified as building blocks to generate the overall EPS model: 1. PL_SAN, which represents the generic power line with the connected transformers. 2 . PR1_SAN and PR2_SAN, which represent the generic protections and the breakers connected to the two extremities of the power line.

3. N_SAN and LCS_SAN, which represent, respectively, a node of the grid (a generator, a load or a substation) and the associated Local Control System. 4. AUTOEV_SAN and RS_SAN, which represent, respectively, the automatic evolution (autoevolution) of EI when an event modifying its state occurs, and the local reconfiguration strategy applied by $L C S$ (function $R S_{1}($ )). 5. RTS_SAN and COMNET_SAN, which represent, respectively, the Regional Telecontrol System RTS, where the regional reconfiguration strategy $R S_{2}()$ is modelled, and the public or private networks (ComNet of Fig. 2).

Then the atomic models are composed and replicated to obtain the composed model representing the EPS region. The different atomic models interact with each other sharing some places of the SAN (common and extended) that represent the parameters or part of the states of the EPS, like the topology of the grid, the susceptance of each line, the initial and the current power of each node of the grid, the initial and the current power flow through each line of the grid, the status of the propagation of a failure or a lightning, the disrupted/failed components, the open lines, etc. Through these interactions, it is also possible to represent the interdependencies between II and EI subsystems and the subsequent cascading or escalation failures caused by malfunctions (either at cyber or electrical levels). 


\section{Models interaction}

While each single model in isolation can be considered as a model of the behaviour of an EPS whose communication infrastructure has been attacked by a DoS, it is quite clear that there are a number of simplifying hypothesis behind. The SWN model assumes that the load shedding command is issued whenever the EI is in a Partial e-outage state, but this is not always the case in reality, it depends on the state of the EI, similarly, the model assumes that the e-restoration can take place or not depending on the number of armed substations, and that any restoration is successful: again this depends on the state of the power grid, that is not included in the model. Nevertheless the modelling of the arming and successive load shedding command by the sentinel, in presence of a DoS, represents quite faithfully the scenario's behaviour.

For what concerns the SAN model there are also a number of discrepancies with respect to the considered scenario: there is no explicit modelling of the Distribution grid and its control. The control is a two level hierarchy (while it is three levels in the scenario). Many of this information are taken into account directly by the reconfiguration function $R S_{2}$ (), for example the reconfiguration can be computed on a limited portion of the grid, thus "emulating" the concept of Area Control Center. The SAN model that has been presented in Section 4 can account for a loss of an electrical component, which is what triggers the computation of a new configuration of the electrical state of the grid through $R S_{2}()$. The SAN model can also account for a failure in the II in the following manner: the $R S_{2}()$ functions is computed on the subset of LCS that are estimated being reachable at the time $R S_{2}()$ is called. Therefore all types of II failures are modelled in terms of the number of LCS components available for a reconfiguration. In the SAN model this number is computed based on a probability of an LCS being reachable or not. The weak point is that this probability should be a model parameter, moreover an important characteristics of the DoS is that its behaviour changes over time, and this should be taken into account in the model.

Fig. 3 depicts the behaviour of the EI and II upon EIfailures (left) and the behaviour of the DoS attack in terms of its severity levels (right), so that a DoS severity level is associated with each event in the time-line.

The first time-line of Fig. 3 depicts a case in which an EI-failure causes the start of the computation of $R S_{2}()$ at time 0 . At time $\Delta T$ the computation terminates, the new reconfiguration is applied. If the reconfiguration was adequate a stable state is reached, if not, some electrical component will disconnect due to the local protections and a new $R S_{2}()$ is computed, leading potentially to a load shedding request. Observe that the reconfiguration success depends also on the DoS attack severity. The second time-line shows

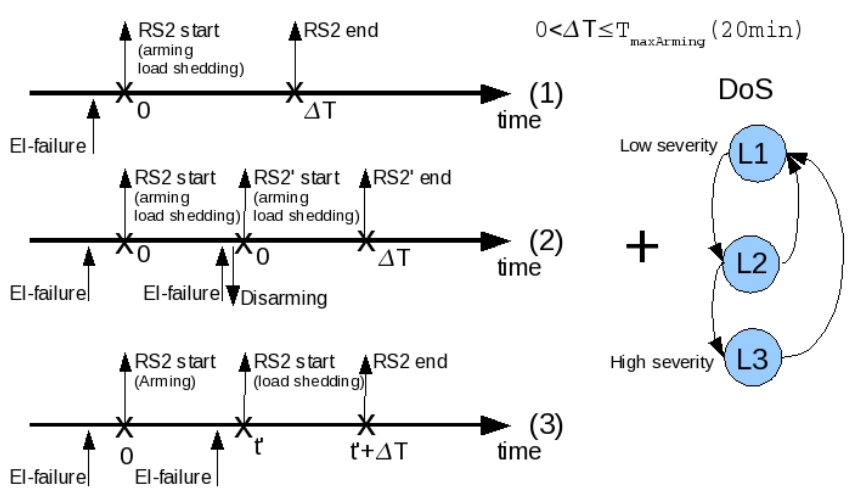

Figure 3: Timed evolution of the EI and II upon EI-failure and the DoS attack behaviour.

instead a case in which between time 0 and $\Delta T$ a second EI failure takes place. In this case the $R S_{2}()$ function is aborted, all the armed LCSs are disarmed, and a new $R S_{2}()$ function is started. The third time-line shows a case where a low severity EI-failure happens followed by a high severity one: the former EI-failure moves the system in alert state and triggers the arming process at time 0 , while the latter one moves the system in alarm state and triggers the load shedding process at time $t^{\prime}$.

The interaction between SWN and SAN takes place precisely on the computation of the number of available LCSs at time $t$ given the arming process started at time 0 , and given an initial DoS severity level. This number is a random variable whose value at time $t$ is distributed according to (prob(NumStation, t|InitDosLevel)). This distribution is computed in isolation on the SWN as the number of armed substations at a finite time horizon $t$ and for an initial DoS severity level. If we consider a behaviour like that depicted in the first two time-lines of Fig. 3, and we consider that the DoS level at time 0 is $L$, then the SWN should compute the distributions at times $\Delta T$ given the initial DoS severity level $L(\operatorname{prob}(*, \Delta T \mid L))$. Instead, if we consider the policy depicted in the last time-line, we should compute the distributions at times $t+\Delta T$ given the initial DoS severity level $L(\operatorname{prob}(*, t+\Delta T \mid L))$. This is due to the fact that the arming process is trigger by the first EI-failure, while the load shedding process by the second EI-failure happened at time $t$. To compute this distribution the SWN model can be significantly simplified (as depicted in Fig.1B), since all aspects concerning the Electrical behaviour (issue of arming and load shedding commands, electrical failure, etc.) are already taken into account by the SAN model. These distributions can be used in the SAN model in two different ways. In the first case, $R S_{2}()$ reconfiguration is computed on $k$ LCSs, then we use $\operatorname{prob}(k, \Delta T \mid L)$ to decide if the reconfiguration induced by $R S_{2}$ () leads the EI in a stable state. In the second case, $R S_{2}()$ reconfiguration is still computed on 
$k$ LCSs, but we use $\operatorname{prob}(*, \Delta T \mid L)$ to compute the number of available LCSs at time $t$ that will be really involved in the reconfiguration given the initial DoS severity level $L$.

Finally we have to highlight that $\operatorname{prob}(*, \Delta T \mid L)$ can be also used to evaluate "a posteriori" the credibility of the performance measures obtained on the SAN model where the $R S_{2}()$ functions are computed on the fixed subset of LCSs that are estimated being reachable at the time $R S_{2}()$.

\section{Numerical Evaluations and Analysis of the Results}

The analyzed electric power grid (Fig. 4) is the IEEE Reliability Test System published in 1999 (RT96) ${ }^{2}$. Here,

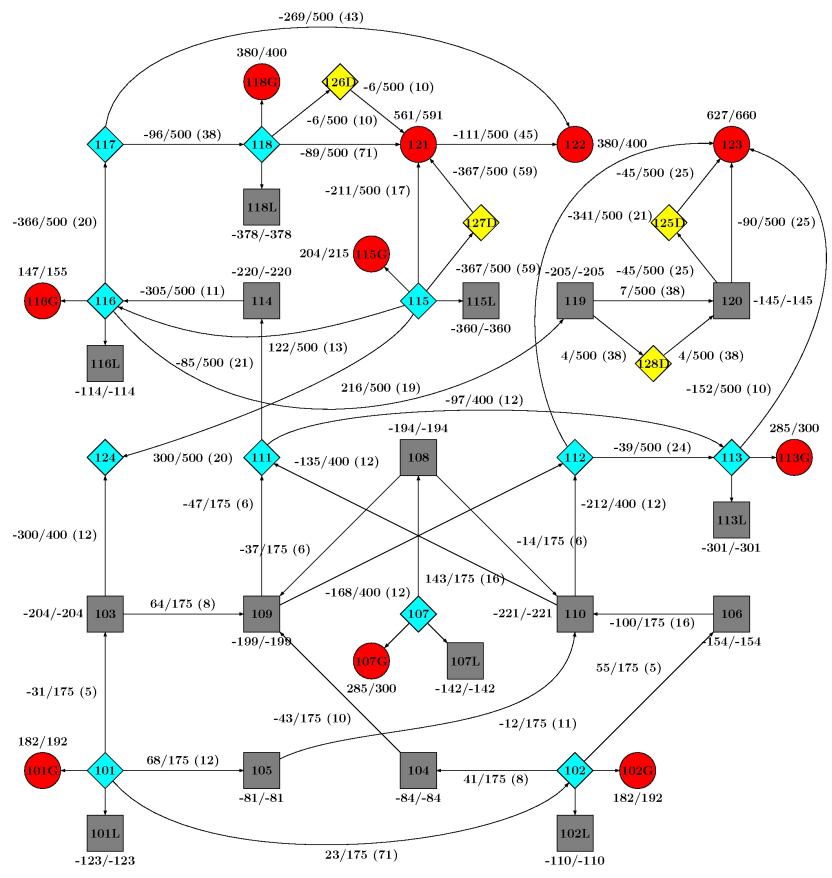

Figure 4: Diagram of the EI grid (generators are circles, loads are squares and substations are diamonds).

a dummy node (with the label "D" associated with the index) is added to represent the lines which are assumed to be on a common right of way or common tower for at least a part of their length. The label associated with the generators represents the initial (active) power and the maximum power that a generator can supply (" $P_{i} / P_{i}^{\max }$ "). The label associated with the loads represents the power demand of a load (" $P_{i}$ "). The label associated with the lines represents the initial power flow through the line, the maximum power flow that each line can carry and the susceptance used to determine the values for the power flow through the lines (" $F_{i j} / F_{i j}^{\max }\left(b_{i j}\right)$ "). In the initial grid setting all the ratios

\footnotetext{
${ }^{2}$ http://www.ee.washington.edu/research/pstca/rts/pg_tcarts.htm
}

$P_{i} / P_{i}^{\max }$ are equal to a fixed value $\alpha=0.95$, called the power grid stress level. At time zero we suppose that one power line is affected by a permanent disruption (e.g., due to a tree fall or a terrorist attack), thus becoming unavailable. The repair time of the failed power lines is fixed to 24 hours. The DoS attack ends after an exponentially distributed time with mean $M T T R^{C N E T}$, and from that time RTS can start computing the $R S_{2}()$ reconfiguration action that will be applied after 10 minutes.

The measure of interest we consider is $P_{U D}(0, t)$, defined as the percentage of the mean power demand that is not met in the interval $[0, t]$ (" $U D$ " stands for 'Unsatisfied Demand'). It is a user-oriented measure of the blackout size and can be obtained as the load shed (i.e., the not served power due to a load shedding) divided by the power demand. In particular, we evaluate the impact on $P_{U D}(0, t)$ of the DoS failure of the communication network when a simultaneous failure of a transmission line occurred, and we make a sensitivity analysis varying the power line that fails at time zero, with $t=4 \mathrm{~d}$.

Now we present some of the results that we obtained through the solution of the overall model previously sketched. A transient analysis has been performed, using the simulator provided by the Möbius tool [10]. For each study we executed a minimum of 2000 simulation runs (batches), and we set the relative accuracy to 0.1 and the confidence level to 0.95 .

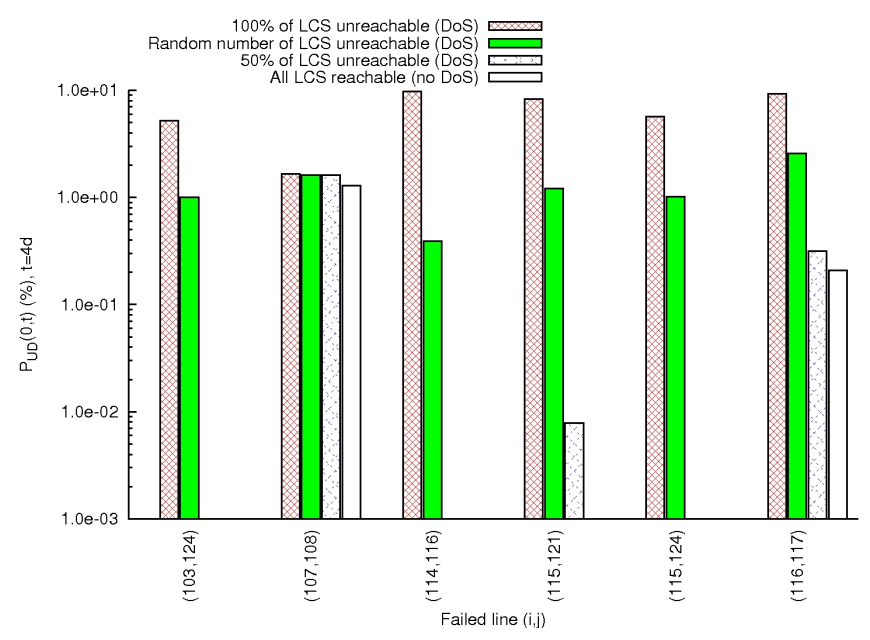

Figure 5: $P_{U D}(0, t)$ for different failed power lines, varying the number of unavailable LCS due to the DoS attack

In Figure 5 we show $P_{U D}(0,4 d)$ for different failed power lines identified by pairs $\left\langle n, n^{\prime}\right\rangle$ of connected nodes, varying the number of LCS that become unavailable due to the DoS attack, for $M T T R^{C N E T}=24$ hours. The first bar considers that all LCS are unavailable, and in this case all the considered power lines are "critical", i.e., their failure induces an unsatisfied power demand. The second bar con- 
siders a random number of unavailable LCS, following distribution computed by the SWN model assuming the initial DoS severity level is "L3" (High severity). In this case, all the considered lines are still identified as critical, but the impact of their failures on $P_{U D}(0,4 d)$ is less significant than in the previous case. The third bar considers that only $50 \%$ of LCS are unavailable, and in this case there are only 3 critical power lines. Finally, the last bar represents the best case where no DoS has occurred and all the LCS are always connected to the network. In this best case only two power lines are "critical". Moreover in this last case, we obtain a significant reduction of $P_{U D}(0,4 d)$ w.r.t. the previous case; for instance $P_{U D}(0,4 d)$ for line $(115,121)$ is 3 orders of magnitude smaller than the previous one considering only $50 \%$ of LCS are unavailable.

\section{Conclusions and Future Work}

In this paper we have shown two different approaches to the modelling and quantification of dependencies between the EI and the II in a EPS. The two models have been instantiated on a specific scenario of cyberattack to the EPS: a DoS attack during a control teleoperation in which DSO and TSO cooperate on a load shedding activity.

The two approaches suffer from a number of limitations and in this paper we have investigated how they can be overcome by a synergic use of the two. The interaction is based on a computation, on the SWN model, of a set of distributions that are then used by the SAN model to characterise the DoS attack behaviour. We performed a preliminary evaluation of the interacting models and additional evaluations are currently running.

An alternative approach could be to extend the SAN model to embed a SAN submodel equivalent to the SWN one: this approach however would significantly increase the simulation time, moreover the efficient SWN specific solution methods [14] could not be applied.

\section{References}

[1] M. Anghel, K. A. Werley, and A. E. Motter. Stochastic model for power grid dynamics. In 40th Hawaii Int. Conference on System Sciences (CD-ROM), pages 113-122, Waikoloa, Big Island, Hawaii, January 2007. IEEE.

[2] S. Baarir, M. Beccuti, D. Cerotti, M. D. Pierro, S. Donatelli, and G. Franceschinis. The GreatSPN Tool: Recent Enhancements. ACM Performance Evaluation Review Spec. Issue on Tools for Perf. Eval., 36:(4): 4-9, 2009.

[3] U. Beyer and F. Flentge. The ISE Metamodel of Critical Infrastructures. In Critical Infrastructure Protection, volume 253/2007 of IFIP International Federation for Information Processing, pages 332-336. Springer Boston, 2007.

[4] J. Chen, J. S. Thorp, and I. Dobson. Cascading dynamics and mitigation assessment in power system disturbances via a hidden failure model. Electrical Power and Energy Systems, 27(4):318-326, 2005

[5] S. Chiaradonna, F. Di Giandomenico, and P. Lollini. Evaluation of critical infrastructures: Challenges and viable approaches. In Architecting Dependable Systems V, volume 5135 of $L N C S$, pages 52-77. Springer Verlag, 2008.

[6] S. Chiaradonna, F. Di Giandomenico, and P. Lollini. Interdependency analysis in electric power systems. In 3rd International Workshop on Critical Information Infrastructures Security (CRITIS 08), pages 63-74, 2008.

[7] S. Chiaradonna, P. Lollini, and F. Di Giandomenico. On a modeling framework for the analysis of interdependencies in electric power systems. In IEEE/IFIP 37th Int. Conference on Dependable Systems and Networks (DSN 2007), pages 185-195, Edinburgh, UK, June 2007.

[8] Cigré Task Force C2.02.24. Defence plan against extreme contingencies. 2007.

[9] CRUTIAL. European Project CRUTIAL - critical utility infrastructural resilience (contract n. 027513). http://crutial.cesiricerca.it.

[10] D. Daly, D. D. Deavours, J. M. Doyle, P. G. Webster, and W. H. Sanders. Möbius: An extensible tool for performance and dependability modeling. In B. R. Haverkort, H. C. Bohnenkamp, and C. U. Smith, editors, 11th International Conference, TOOLS 2000, volume 1786 of LNCS, pages 332-336. Springer Verlag, 2000.

[11] I. Dobson, B. A. Carreras, V. Lynch, and D. E. Newman. An initial model for complex dynamics in electric power system blackouts. In 34th Hawaii Int. Conference on System Sciences (CD-ROM), Maui, Hawaii, January 2001. IEEE.

[12] S. Donatelli. Dependent automata for the modelling of dependencies. In 3rd International Workshop on CRITIS'08, pages 367-374, 2008.

[13] G. Dondossola et al. On EPS-ICT interdependencies in the testbeds. Workpackage 3 Deliverable D17, CRUTIAL consortium. 2009

[14] G. Chiola, C. Dutheillet, G. Franceschinis, and S. Haddad. Stochastic Well-formed Coloured nets for symmetric modelling applications. IEEE Transactions on Computers, 42:(11): 1343 - 1360, 1993.

[15] IRRIIS. European Project IRRIIS - integrated risk reduction of information-based infrastructure systems. http://www.irriis.org/.

[16] M. Kaaniche et al. The CRUTIAL modeling framework. Workpackage 2 Deliverable D16, CRUTIAL consortium. 2009.

[17] J.-C. Laprie, K. Kanoun, and M. Kaniche. Modeling interdependencies between the electricity and information infrastructures. In SAFECOMP-2007, volume 4680 of LNCS, pages 54-67. Springer Verlag, 2007.

[18] W. H. Sanders and J. F. Meyer. Stochastic activity networks: Formal definitions and concepts. In Lectures on Formal Methods and Performance Analysis, volume 2090 of LNCS, pages 315-343. Springer Verlag, 2001.

[19] A. Usov and C. Beyel. Simulating interdependent Critical Infrastructures with SimCIP. European CIIP Newsletter, 4(3), 2008. 